\title{
On the extension of inverse Lindley distribution
}

\author{
Vikas Kumar Sharma $^{1}$, Pragya Khandelwal ${ }^{2}$ \\ Department of Mathematics, Institute of Infrastructure Technology, Research and \\ Management (IITRAM), Maninagar East, Ahmedabad, India \\ Department of Statistics \\ Central University of Rajasthan, Bandersindri, Ajmer, India
}

\begin{abstract}
In this paper, we proposed another extension of inverse Lindley distribution, called extended inverse Lindley and studied its fundamental properties such as moments, inverse moments, mean deviation, stochastic ordering and entropy. The flexibility of the proposed distribution is shown by studying monotonicity properties of density and hazard functions. It is shown that the distribution belongs to the family of upside-down bathtub shaped distributions. Maximum likelihood estimators along with asymptotic confidence intervals are constructed for estimating the unknown parameters. An algorithm is presented for random number generation form the distribution. The property of consistency of MLEs has been verified on the basis of simulated samples. The applicability of the extended inverse Lindley distribution is illustrated by means of real data analysis.
\end{abstract}

Keywords: Extended inverse Lindley distribution, upside-down bathtub shaped hazard rate, Moments, Maximum likelihood estimation.

\section{Introduction}

In 1958, Prof. D.V. Lindley (Lindley (1958)) investigated a probability distribution in con-text of fiducial statistic as a counter example of Bayesian theory. Later, this distribution is called as the Lindley distribution (LD). Ghitany et al. (2008) discussed the fundamental properties of the LD with application to waiting time data. Mazucheli and Achcar (2011) worked on the Lind-ley distribution applied to competing risks lifetime data. Krishna and Kumar (2011) estimated the parameter of Lindley distribution with progressive Type-II censoring scheme. They also showed that it may be better lifetime model than exponential, lognormal and gamma distribu-tions in some real life situations. Since then the distribution has been widely discussed in various context. Singh and Gupta (2012) have used the Lindley distribution under load sharing sys-tem models. Al-Mutairi et al. (2013) developed the inferential procedure of the stress-strength parameter, when both stress and strength variables follow Lindley distribution.

It may be mentioned here that the Lindley distribution is useful when the data show increas-ing failure rate. This is the property that encourage the use of Lindley distribution in lifetime data analysis over exponential distribution. Although the family of Lindley distributions posses very nice properties and gained great applicability in various disciplines, its applicability may

\footnotetext{
${ }^{*}$ Corresponding author

Email address: vikasstats@rediffmail.com (Vikas Kumar Sharma ${ }^{\mathrm{a}, \mathrm{b}}$ )
} 
be restricted to non-monotone hazard rate data (bathtub and upside down bathtub (UBT) see Sharma et al. (2014a)). Therefore, the LD has been extended to various ageing classes and introduced various generalized class of lifetime distribution based on Lindley distribution. Zakerzadeh and Dolati (2009) introduced three parameters extension of the Lindley distribution. Nadarajah et al. (2011), Ghitany et al. (2013) proposed two parameter generalizations of the Lindley distribution, called as the generalized Lindley and power Lindley distributions. These distributions are generated using the exponentiation and power transformations to the Lindley distribution. Merovci (2013) and Merovci and Elbatal (2014) investigated transmuted Lindley and transmuted Lindley-geometric distributions respectively. The beta-Lindley distribution is introduced by Merovci and Sharma (2014). Statistical and mathematical properties of Kumaraswamy Quasi Lindley and Kumaraswamy Lindley distributions are discussed by Elbatal and Elgarhy (2013) and Akmakyapan and Kadlar (2014) respectively. The exponentiated power Lindley distribution is introduced by Ashour and Eltehiwy (2015). The generalized Poisson-Lindley and another extension of the Lindley distribution are discussed by Mahmoudi and Zakerzadeh (2010) and Oluyede and Yang (2015).

In the references cited above, authors mainly focused on the estimation of increasing, decreasing and bathtub shaped failure rates data. Nobody has paid attention to the modelling of the upside down bathtub data. Recently, Sharma et al. (2015) investigated inverted version of the Lindley distribution that has UBT shaped failure rate. The inverse Lindley distribution (ILD) is defined by the following probability density function (pdf)

$$
f(x ; \theta)=\left(\frac{\theta^{2}}{1+\theta}\right)\left(\frac{1+x}{x^{3}}\right) e^{-\theta / x}, x>0, \theta>0 .
$$

The above density (1) can be rewrite as

$$
f(x)=p f_{1}(x)+(1-p) f_{2}(x),
$$

where, $p=\frac{\theta}{1+\theta}, f_{1}(x)=\theta x^{-2} e^{-\theta / x}, \quad x>0, \theta>0$ and $f_{2}(x)=\frac{\theta^{2}}{\Gamma 2} x^{-3} e^{-\theta / x}, x>0, \theta>0$.

Thus, inverse Lindley distribution is a two component mixture of inverse exponential distribution and special case of inverse gamma distribution. Sharma et al. (2015) discussed the properties of inverse Lindley distribution with application to stress strength reliability analysis. Sharma et al. (2014b) introduced two parameter extension of inverse Lindley distribution using power transformation to inverse Lindley random variable. Recently, Alkarni (2015) proposed three parameter inverse Lindley distribution with application to maximum flood level data.

In this article, we proposed another extension of inverse Lindley distribution which offers more flexibility with a effective shape parameter. The pdf of the extension is defined by

$$
f(x ; \alpha, \theta)=p \text { InverseGamma }(\alpha-1, \theta)+(1-p) \text { InverseGamma }(\alpha, \theta), \alpha>1, \theta>0,
$$

where $p=\frac{\theta}{1+\theta}$, and InverseGamma $(\alpha, \theta)$ stands for inverse gamma distribution with shape parameter $\alpha$ and scale parameter $\theta$ i.e. $f(x)=\frac{\theta^{\alpha}}{\Gamma \alpha} \frac{e^{-\frac{\theta}{x}}}{x^{(\alpha+1)}}, x>0, \theta>0, \alpha>0$. The pdf of the EILD is given by

$$
f(x ; \alpha, \theta)=\frac{\theta^{\alpha}}{(1+\theta) \Gamma(\alpha)} \frac{(1+x(\alpha-1))}{x^{\alpha+1}} e^{-\theta / x}, x>0, \theta>0, \alpha>1 .
$$

The corresponding cumulative distribution function of (3) is given by

$$
F(x ; \alpha, \theta)=p \text { CDFInverseGamma }(\alpha-1, \theta)+(1-p) \text { CDFInverseGamma }(\alpha, \theta), \alpha>1, \theta>0,
$$


where, CDFInverseGamma $(\alpha, \theta)=\frac{\Gamma(\theta / \mathrm{x}, \alpha)}{\Gamma \alpha} \quad, \Gamma(\mathrm{t}, \alpha)=\int_{0}^{\infty} \mathrm{x}^{\alpha-1} \mathrm{e}^{-\mathrm{x}} \mathrm{dx}$ and $\Gamma(\alpha)=\oint^{\infty} \mathrm{t}^{(\mathrm{s}-1)} \mathrm{e}^{-\mathrm{t}} \mathrm{dt}$.

We call the distribution in (3) as the extended inverse Lindley distribution and denote the density as $\operatorname{EILD}(\alpha, \theta)$. Note that if $\mathrm{X}$ follows $\operatorname{EILD}(\alpha, \theta)$, then $\mathrm{Y}=\mathrm{X}^{-1}$ follows the generalized Lindley distribution by Abouammoha et al. (2015).

Rest of the article is organized in the following sections. The distributional properties such as moments, skewness, kurtosis, mean deviation, stochastic ordering and Reyni entropy are investigated in section 1. The maximum likelihood estimation along with asymptotic distribution is discussed in section 2. Section 3 consists algorithm for random sample generation form the EILD. Simulation study has also been carried out study the performances of the MLEs. A set of real data is used for illustration purposes in section 4. The paper is concluded in section 5 .

\section{Distributional properties}

In this section, we discuss the fundamental properties of the EILD. Mainly the properties to be discussed include measures of central tendency, dispersion and shapes of the frequency distribution from EILD. First, we study the shapes of the pdf and hazard function in the following section.

\subsection{Shapes of Density and Hazard functions}

The pdf of extended inverse Lindley distribution is given by

$$
f(x ; \alpha, \theta)=\frac{\theta^{\alpha}}{(1+\theta) \Gamma(\alpha)} \frac{(1+x(\alpha-1))}{x^{\alpha+1}} e^{-\theta / x}, x>0, \theta>0, \alpha>1 .
$$

The first derivative of the pdf with respect to $\mathrm{x}$ is readily obtained as

$$
\mathrm{f}^{\prime}(\mathrm{x})=\frac{\theta^{\alpha}}{\left(\theta-(\alpha-\theta+1) \mathrm{x}-\alpha \mathrm{x}^{2}\right)}\left(\mathrm{e}^{-\theta / \mathrm{x}} .\right.
$$

By putting $\mathrm{f}^{\prime}(\mathrm{x})=0$, we get the mode of EILD which is given by

$$
\mathrm{x}_{\text {mode }}=\frac{-(\alpha \theta-\theta+\alpha+1) \pm \sqrt{(\alpha \theta-\theta+\alpha+1)^{2}+4\left(\alpha \theta-\alpha^{2} \theta\right)}}{2\left(\alpha-\alpha^{2}\right)} .
$$

For various shapes of the pdf of EILD, see Figure 1a. The survival function of the density is given by $\mathrm{S}$ $(\mathrm{x})=1-\mathrm{F}(\mathrm{x})$. The survival function of EILD have been plotted in Figure $1 \mathrm{~b}$ for various combination of $\alpha$ and $\theta$.

The hazard function of extended inverse Lindley distribution is given by

$$
\mathrm{h}(\mathrm{x})=\frac{\theta^{\alpha} \Gamma(\alpha) \mathrm{e}^{-(\theta / \mathrm{x})}(1+\mathrm{x}(\alpha-1))}{\mathrm{x}^{\alpha-1}[(\theta+1) \Gamma \alpha-\{\gamma(\alpha, \theta / \mathrm{x})+\theta(\alpha-1) \gamma(\alpha-1, \theta / \mathrm{x})\}]} .
$$

The hazard function of EILD for various choices of $\alpha$ and $\theta$ is shown in Figure 2 indicates that the hazard function is upside down bathtub shaped. 

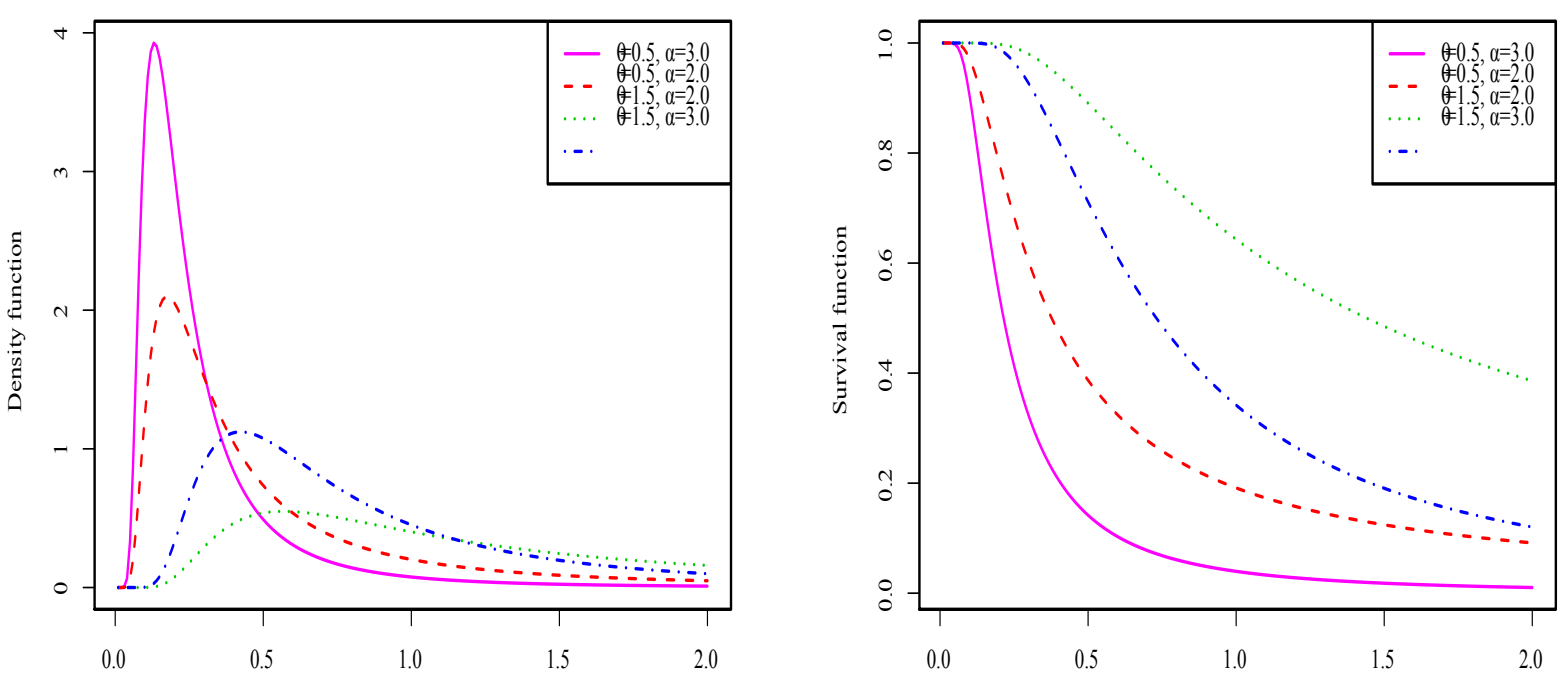

Density function.

Survival function.

Figure 1: Density and survival functions of $\operatorname{EILD}(\alpha, \theta)$.

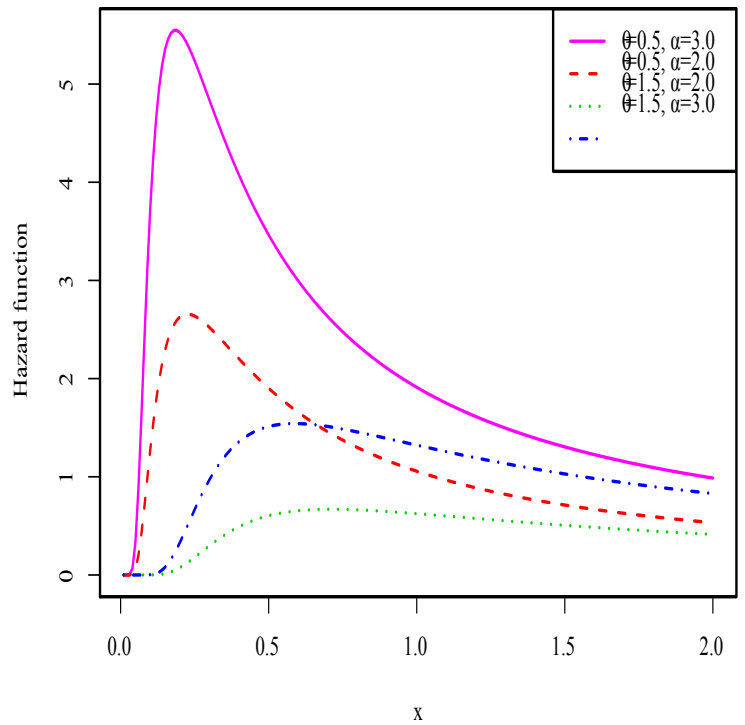

Figure 2: The hazard function of the $\operatorname{EILD}(\alpha, \theta)$. 
2.2. Moments, inverse moments and associated Measures

The $\mathrm{r}^{\text {th }}$ moment can be defined by,

$$
E\left[X^{r}\right]=\int_{0}^{\infty} x^{r} f(x ; \alpha, \theta) d x .
$$

On putting the density function, we get

$$
E\left[X^{r}\right]=\int_{0}^{\infty} x^{r} \frac{\theta^{\alpha}}{(1+\theta) \Gamma(\alpha)} \frac{(1+x(\alpha-1))}{x^{\alpha+1}} e^{-\theta / x} d x
$$

Using the definition of inverse gamma, we obtain

$$
\mathrm{E}\left[\mathrm{X}^{\mathrm{r}}\right]=\frac{\underline{\theta}^{\mathrm{r}}}{[(\theta+1)(\alpha-1)-\mathrm{r}] \Gamma(\alpha-\mathrm{r}-1)}(\theta+
$$

The $\mathrm{r}^{\text {th }}$ inverse moment $\mathrm{E} \quad\left[\frac{1}{\mathrm{X}^{\mathrm{r}}}\right]$ can be obtained by

$$
E\left[\frac{1}{X^{r}}\right]=\int_{0}^{\infty} \frac{1}{x^{r}} f_{X}(x) d x .
$$

After substituting the pdf of the ILD, we have

$$
\mathrm{E}\left[\frac{1}{\mathrm{X}^{\mathrm{r}}}\right]=\int_{0}^{\infty} \frac{1}{\mathrm{X}^{\mathrm{r}}} \frac{\theta^{\alpha}}{(1+\theta) \Gamma(\alpha)} \frac{(1+\mathrm{x}(\alpha-1))}{\mathrm{x}^{\alpha+1}} \mathrm{e}^{-\theta / \mathrm{x}} \mathrm{dx} .
$$

On some simplifications, we get

$$
\mathrm{E}\left[\frac{1}{\mathrm{X}^{\mathrm{r}}}\right]=\frac{[(\alpha-1)(1+\theta)+\mathrm{r}] \Gamma(\alpha+\mathrm{r}-1)}{+1) \theta^{\mathrm{r}} \Gamma(\alpha)}(\theta .
$$

The harmonic mean of the EILD can be computed using the first inverse moment as 1/E The first $\left[\frac{1}{\bar{X}}\right]$. four moments of EILD are given by

$$
\begin{gathered}
\mu{ }_{1}=\mathrm{E}[\mathrm{x}]=\frac{\theta[(\theta+1)(\alpha-1)-1]}{(\theta+1)(\alpha-1)(\alpha-2)}, \alpha>2, \\
\mu{ }_{2}=\frac{\theta^{2}[(\theta+1)(\alpha-1)-2]}{(\theta+1)(\alpha-1)(\alpha-2)(\alpha-3)}, \alpha>3, \\
\mu_{3}=\frac{\theta^{3}[(\theta+1)(\alpha-1)-3]}{(\theta+1)(\alpha-1)(\alpha-2)(\alpha-3)(\alpha-4)}, \alpha>4, \\
\mu \prime_{4}=\frac{\theta^{4}[(\theta+1)(\alpha-1)-4]}{(\theta+1)(\alpha-1)(\alpha-2)(\alpha-3)(\alpha-4)(\alpha-5)}, \alpha>5 .
\end{gathered}
$$

The variance of the EILD can be computed using the following relation,

$$
\sigma^{2}=\mu_{2}{ }^{\prime}-\mu_{1}{ }^{2}
$$




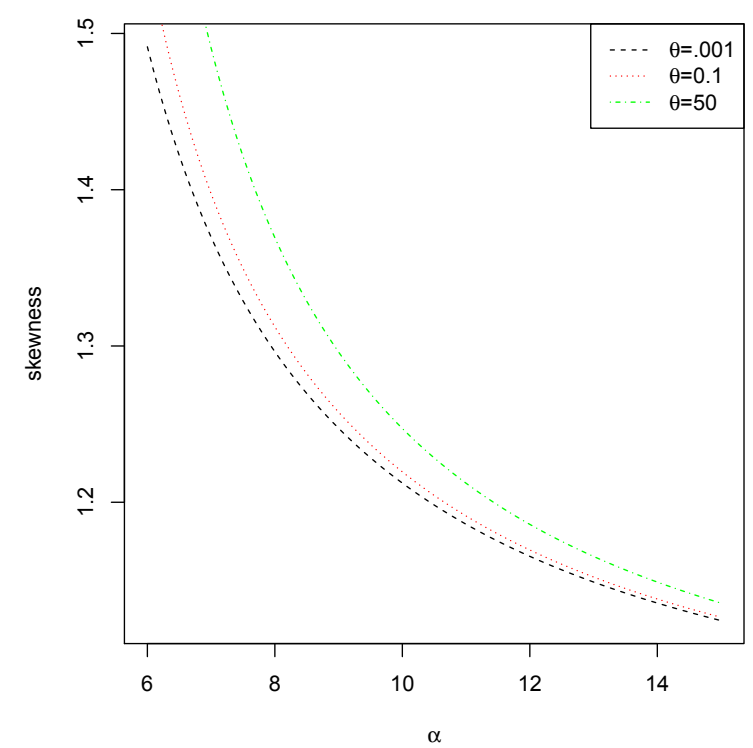

(a) Coefficients of skewness.

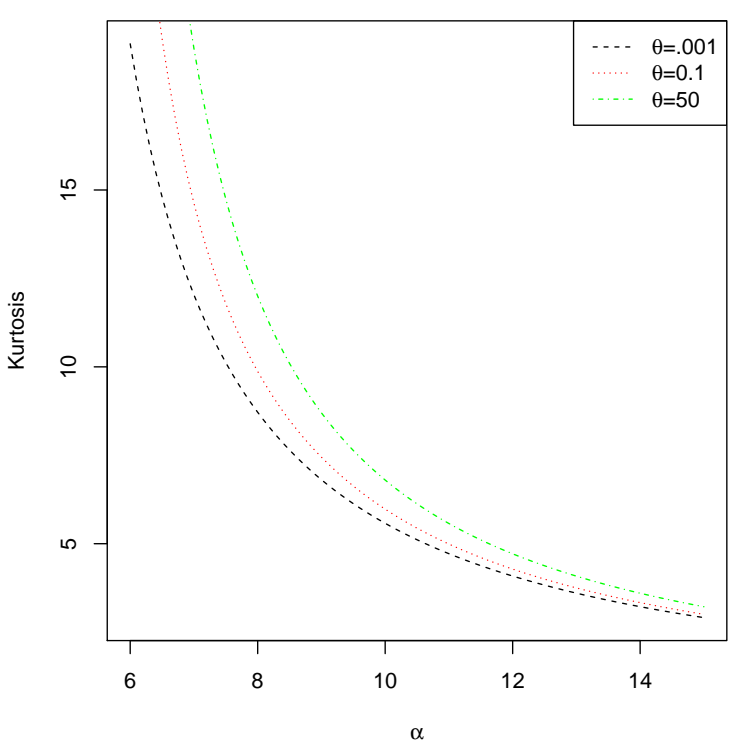

(b) Coefficients of kurtosis.

Figure 3: Plots for skewness and kurtosis of $\operatorname{EILD}(\alpha, \theta)$.

The variance of the EILD random variable is given by

$$
\sigma^{2}=\frac{\left[(\theta+1)^{2}(\alpha-1)^{2}-2(\theta+1)(\alpha-1)-(\alpha-3)\right]}{(\theta+1)^{2}(\alpha-1)^{2}(\alpha-2)^{2}(\alpha-3)}, \quad \alpha>3 .
$$

The coefficients of the skewness and kurtosis measures can now be computed from the following expression,

$$
\begin{gathered}
\operatorname{skewness}\left(\gamma_{1}\right)=\frac{\left[\mu_{3} \prime-3 \mu_{1} \mu_{2}{ }^{\prime}+2\left(\mu_{1} \prime\right)^{3}\right]}{\left[\mu_{2} \prime-\left(\mu_{1}\right)^{2}\right]^{3 / 2}}, \alpha>3 \\
\operatorname{kurtosis}\left(\gamma_{2}\right)=\beta_{2}-3
\end{gathered}
$$

where,

$$
\beta_{2}=\frac{\left[\mu_{4}^{\prime}-4 \mu_{1} \mu_{3} \prime+6 \mu_{1}^{2} \mu_{2} \prime-3 \mu_{1}^{4}\right]}{\left[\mu_{2} \prime-\left(\mu_{1}\right)^{2}\right]^{2}}, \quad \alpha>4 .
$$

The skewness for EILD is shown in Figure 3a for various choices of $\alpha$ and $\theta$. From the figure it can be observed that the $\gamma_{1}>0$ so the EILD is positively skewed. The kurtosis is shown in Figure $3 \mathrm{~b}$ for various values of $\alpha$ and $\theta$. It can be observed from the figure that $\gamma_{2}>0$. EILD is more peaked than normal curve so it is leptokurtic.

\subsection{Mean Deviation and Median Deviation}

Lemma 1. Let

$$
I(x ; A)=\int_{A}^{\infty} x f(x) d x
$$

then

$$
I(x ; A)=\frac{\theta}{(1+\theta) \Gamma \alpha}\left[\Gamma\left(\frac{\theta}{A}, \alpha-1\right)+\theta(\alpha-1) \Gamma\left(\frac{\theta}{A}, \alpha-2\right)\right], \quad \alpha>2 .
$$


Proof Consider the given integral,

$$
I(x ; A)=\int_{\alpha}^{\infty} x f(x) d x=\frac{\theta^{\alpha}}{(1+\theta) \Gamma \alpha} \int_{\alpha}^{\infty} \frac{(1+x(\alpha-1))}{x^{\alpha}} e^{-\theta / x} d x .
$$

On some simplifications, we get $\theta$

$$
\mathrm{I}(\mathrm{x} ; \mathrm{A})=\frac{}{(1+\theta) \Gamma \alpha}[\Gamma(\underline{\theta}, \alpha-1)+\theta(\alpha-1) \Gamma(\stackrel{\theta}{\AA}, \alpha-2)] .
$$

The mean deviation about mean and median are defined by,

$$
M_{1}(x)=\int_{0}^{\infty}(x-\mu) f_{X}(x) d x=M_{1}(x)=2 \mu F(\mu)+2 \quad \int_{\mu}^{\infty} x f_{X}(x) d x-\mu-1,
$$

and

$$
\mathrm{M}_{2}(\mathrm{x})=\int_{0}^{\infty}(\mathrm{x}-\mathrm{m}) \mathrm{f}_{\mathrm{X}}(\mathrm{x}) \mathrm{dx}=\mathrm{M}_{2}(\mathrm{x})=2 \quad \int_{\mathrm{h}}^{\infty} \mathrm{x} \mathrm{f}_{\mathrm{X}}(\mathrm{x}) \mathrm{dx}-1
$$

respectively, where $\mu$ and $\mathrm{m}$ represent the mean and median of the EILD. Using the Lemma 1, we get

$$
\mathrm{M}_{1}(\mathrm{x})=2 \mu \mathrm{F}(\mu)+2 \mathrm{I}(\mathrm{x} ; \mu)-\mu-1
$$

and

$$
\mathrm{M}_{2}(\mathrm{x})=2 \mathrm{I}(\mathrm{x} ; \mathrm{m})-1
$$

2.4. Stochastic ordering

A random variable $X$ is said to be stochastically greater than $Y$ if $F_{X}(x)<=F_{Y}(x)$ for all $\mathrm{X}$. In the similar way, $\mathrm{X}$ is said to be stochastically greater than $\mathrm{Y}$ in the

- hazard rate order $\left(\mathrm{Y} \leq_{\mathrm{hr}} \mathrm{X}\right)$ if $\mathrm{h}_{\mathrm{X}}(\mathrm{x}) \leq \mathrm{h}_{\mathrm{Y}}(\mathrm{x})$ for all $\mathrm{x}$.

- mean residual life order $\left(\mathrm{Y} \leq_{\mathrm{mrl}} \mathrm{X}\right)$ if $\mathrm{m}_{\mathrm{X}}(\mathrm{x}) \leq \mathrm{m}_{\mathrm{Y}}(\mathrm{x})$ for all $\mathrm{x}$.

- likelihood ratio order $\left(\mathrm{Y} \leq_{\mathrm{lr}} \mathrm{X}\right)$ if $\frac{\mathrm{f}}{\mathrm{Y}}_{\mathrm{Y}} \frac{\mathrm{x}}{\mathrm{x}}_{\mathrm{f}}$ (x) is an increasing function of $\mathrm{x}$.

Shaked and Shanthikumar (1994) have stated the results for stochastic ordering of distribution as follows:

$$
\mathrm{X} \leq_{\mathrm{lr}} \mathrm{Y} \Rightarrow \mathrm{X} \leq_{\mathrm{hr}} \mathrm{X} \underset{\mathrm{X} \leq_{\mathrm{st}}^{\Downarrow}}{ } \quad \Rightarrow \mathrm{X} \leq_{\mathrm{mrl}} \mathrm{Y}
$$

Following the above relations, to show the above ordering in hr, mlr and lr, it is suffix to show the ordering in likelihood only.

Proposition 1: Let $\mathrm{X}$ and $\mathrm{Y}$ are two independent random variables follow the extended inverse Lindley distribution with shape parameters $\alpha_{1}$ and $\alpha_{2}$ and scale parameters $\theta_{1}$ and $\theta_{2}$ respectively. If $\theta_{2} \geq \theta_{1}$, then $(\mathrm{Y} \leq \mathrm{X})$ and if $\alpha_{1} \geq \alpha_{2}$, then $(\mathrm{Y} \leq \mathrm{X})$ for all $\mathrm{x}$, while other parameters kept fixed and same. 
Proof It is given that $\mathrm{Y} \sim \operatorname{EILD}\left(\alpha_{1}, \theta_{1}\right)$ and $\mathrm{X} \sim \operatorname{EILD}\left(\alpha_{2}, \theta_{2}\right)$. Then the likelihood ratio is given by,

$$
\frac{f_{X}(x)}{f_{Y}(x)}=\frac{\theta_{2}^{\alpha_{2}} e^{-\theta_{2} / x}\left(\theta_{1}+1\right) x^{\alpha_{1}-1}\left\{1+x\left(\alpha_{2}-1\right)\right\}}{\theta_{1}^{\alpha_{1}} e^{-\theta_{1} / x}\left(\theta_{2}+1\right) x^{\alpha_{2}-1}\left\{1+x\left(\alpha_{1}-1\right)\right\}}
$$

Now for $\alpha_{1}=\alpha_{2}=\alpha$, we have

$$
\frac{f_{X}(x)}{f_{Y}(x)}=\frac{\left(1+\theta_{1}\right)}{\left(1+\theta_{2}\right)}\left(\frac{\theta_{2}}{\theta_{1}}\right)^{\alpha} \exp \left[\frac{-\left(\theta_{2}-\theta_{1}\right)}{x}\right]
$$

and

$$
\log \left(\frac{f_{X}(x)}{f_{Y}(x)}\right)=\log \left(1+\theta_{1}\right)-\log \left(1+\theta_{2}\right)+\alpha \log \left(\frac{\theta_{2}}{\theta_{1}}\right)-\frac{\left(\theta_{2}-\theta_{1}\right)}{x}
$$

On differentiating, we get

$$
\frac{\delta}{\delta x}\left\{\log \left(\frac{f_{X}(x)}{f_{Y}(x)}\right)\right\}=\frac{\left(\theta_{2}-\theta_{1}\right)}{x^{2}} .
$$

We can easily find that the function above is increasing in $x$ for $\theta_{2} \geq \theta_{1}$. Which means that $X$ is stochastically greater than $Y$ with respect to likelihood ratio if $\theta_{2} \geq \theta_{1}$ and $\alpha_{1}=\alpha_{2}=\alpha$.

If $\theta_{1}=\theta_{2}=\theta$, the likelihood ratio becomes,

$$
\frac{f_{X}(x)}{f_{Y}(x)}=\left(\frac{\theta}{x}\right)^{\alpha_{2}-\alpha_{1}} \frac{\Gamma \alpha_{2}}{\Gamma \alpha_{1}} \frac{\left[1+x\left(\alpha_{2}-1\right)\right]}{\left[1+x\left(\alpha_{1}-1\right)\right]},
$$

which is increasing in $x$ for $\alpha_{2} \leq \alpha_{1}$. Clearly, for $\theta_{1}=\theta_{2}=\theta, X$ is stochastically greater than $Y$ with respect to likelihood ratio if $\alpha_{2} \leq \alpha_{1}$.

\subsection{Renyi Entropy}

An entropy is a measure of variation of the uncertainty in the distribution of a random variable X. For a probability distribution, the expression of the Renyi entropy (Renyi (1961)) is defined by,

$$
e(\gamma)=\frac{1}{1-\gamma} \log \int f^{\gamma}(x) d x
$$

where, $\gamma>0$ and $\gamma=1$. Substituting (4) in (7) and after some simplifications, we have

$$
e(\gamma)=\frac{1}{1-\gamma} \log \left\{\frac{\theta^{(\alpha+1)(1-\gamma)} \Gamma(\gamma(\alpha+1)-2)(\gamma \alpha+\gamma-2+\alpha \theta \gamma-\theta \gamma)}{(1+\theta) \Gamma(\alpha) \gamma^{\gamma(\alpha+1)-1}}\right\}
$$

\section{Maximum likelihood estimation}

Suppose $X_{1}, X_{2}, X_{3}, \ldots, X_{n}$ are independently and identically distributed random variables of size $n$ from the extended inverse Lindley distribution. The likelihood function based on observed sample $\underline{\mathrm{X}}=\left\{x_{1}, x_{2}, x_{3}, \ldots, x_{n}\right\}$, is defined as

$$
L=\frac{\theta^{n \alpha}}{(1+\theta)^{n}(\Gamma \alpha)^{n}} \prod_{i=1}^{n} \frac{\left(1+x_{i}(\alpha-1)\right)}{x_{i}^{\alpha+1}} e^{-\sum_{i=1}^{n} \frac{\theta}{x_{i}}} .
$$


The log-likelihood function corresponding to (9) is given by

$\log \mathrm{L}=\mathrm{n} \alpha \log \theta-\mathrm{n} \log (1+\theta)-\mathrm{n} \log (\Gamma \alpha)+\quad \sum_{-1}^{\mathrm{n}} \log \left(1+\mathrm{x}_{\mathrm{i}}(\alpha-1)\right)-(\alpha+1) \quad \sum_{\mathrm{i}}^{\mathrm{n}} \log \left(\mathrm{x}_{\mathrm{i}}\right)-\sum_{\mathrm{i}=4}^{\mathrm{n}} \frac{\theta}{\mathrm{x}_{\mathrm{i}}}$.

The maximum likelihood estimates (MLEs) $\hat{\alpha}$ and $\hat{\theta}$ of $\alpha$ and $\theta$, respectively can be obtained as the simulation solution of the following log-likelihood equations,

$$
\begin{gathered}
\frac{\delta \log \mathrm{L}}{\delta \alpha}=\mathrm{n} \log \theta-\mathrm{n}(\alpha)+\sum_{+}^{\mathrm{n}} \frac{\mathrm{x}_{\mathrm{i}}}{\left(1+\mathrm{x}_{\mathrm{i}}(\alpha-1)\right)}-\sum_{+}^{\mathrm{n}} \log \mathrm{x}_{\mathrm{i}}=0, \\
\frac{\delta \log \mathrm{L}}{\delta \theta}=\frac{\mathrm{n} \alpha}{\theta}-\frac{\mathrm{n}}{(1+\theta)}-\sum_{-1}^{\mathrm{n}} \frac{1}{\mathrm{x}_{\mathrm{i}}}=0
\end{gathered}
$$

where $(\alpha)=\frac{\delta \log \Gamma \alpha}{\delta \alpha}$. Using equation (12), the MLE of $\theta$ can be obtained in terms of $\alpha$ as

$$
\hat{\theta}(\alpha)=\frac{-(S-\alpha+1)+\sqrt{(S-\alpha+1)^{2}+4 S \alpha}}{2 S}
$$

where, $\mathrm{S}=\frac{1}{\mathrm{n}} \sum_{=1}^{\mathrm{n}} \frac{1}{\mathrm{x}_{\mathrm{i}}}$.

The MLE of $\alpha$ can be uniquely determined by solving the following non-linear equation,

$$
n \log \hat{\theta}(\alpha)-n \zeta(\alpha)+\sum_{i}^{n} \frac{x_{i}}{\left(1+x_{i}(\alpha-1)\right)}-\sum_{1}^{n} \log x_{i}=0 .
$$

Note that the equation (13) cannot be solved analytically. Therefore, we need numerical iteration technique to solve the equation. Here, we use Newton-Raphson Method for solving the non-linear equations.

\subsection{Approximate confidence intervals}

Obtaining the exact confidence intervals for $\alpha$ and $\theta$ is not an easy task since the MLE's of $\alpha$ and $\theta$ are not in closed forms. We, therefore, can use the asymptotic behaviour of the maximum likelihood estimator to obtained the asymptotic confidence intervals for thedel parameters.

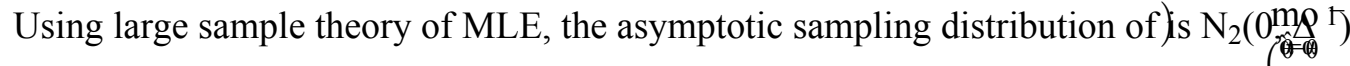

where $(\hat{\alpha}, \hat{\theta})$ are the MLEs of $(\alpha, \theta)$, and $\Delta$ is the observed Fisher's information matrix. The elements of $\Delta$ are given by

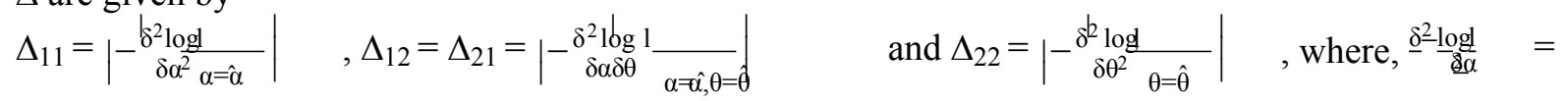

$$
\begin{aligned}
& \mathrm{n} /(\alpha)-\sum_{i=1}^{\mathrm{n}} \frac{\mathrm{x}_{\mathrm{i}}^{2}}{\left[1+\mathrm{x}_{\mathrm{i}}(\alpha-1)\right]^{2}}, \frac{\delta^{2} \log 1}{\delta \alpha \delta \theta}=\frac{\mathrm{n}}{\theta}, \frac{\delta^{2} \log }{\delta \theta}=\frac{\mathrm{n} \alpha}{\mathfrak{Z} \theta}+\frac{\mathrm{n}}{(1+\theta)^{2}} \text {. }
\end{aligned}
$$

The estimated variance co ariance matrix of the parameters $\alpha$ and $\theta$ can be calculated by inverting $\Delta$ as follows $\mathrm{V}^{-1}=\left\{\begin{array}{c}\widehat{\operatorname{var}(\alpha)} \frac{\operatorname{cov}(\alpha, \theta)}{\operatorname{cov}(\theta, \alpha)}(\operatorname{var}(\theta)\end{array}\right\}=\left\{\begin{array}{ll}\Delta_{11} & \Delta_{12} \\ \Delta_{21} & \Delta_{22}\end{array}\right\}^{-1}$.

The diagonal elements $\widehat{\operatorname{var}(\alpha)}$ and $\widehat{\operatorname{var}(\theta)}$ of this matrix are the asymptotic variances of the estimators of $\alpha$ and $\theta$, respectively. Thus, the asymptotic $100(1-) \%$ confidence intervals for $\alpha$ and $\theta$ are given by 


$$
\left\{\begin{array}{cc}
\hat{\alpha}_{L}=\widehat{\alpha}-Z_{\psi / 2} \sqrt{\widehat{\operatorname{var}(\alpha)}}, \quad \hat{\alpha}_{U}=\hat{\alpha}+Z_{\psi / 2} \sqrt{\widehat{\operatorname{var}(\alpha)}} \\
\hat{\theta}_{L}=\hat{\theta}-Z_{\psi / 2} \sqrt{\widehat{\operatorname{var}(\theta)}}, \quad \hat{\theta}_{U}=\hat{\theta}+Z_{\psi / 2} \sqrt{\operatorname{var}(\theta)}
\end{array}\right\}
$$

where, $Z_{\psi / 2}$ is the upper $100 \times(\psi / 2)^{t h}$ percentile of a standard normal distribution.

\section{Simulation Study}

In this section, we investigate the properties of the MLEs of $\alpha$ and $\theta$ with respect to sample size $n$. For this purpose, we need to simulate the random sample from the EILD. Since the EILD is two component mixture of inverse gamma distributions, we can use the definition of the mixture distribution for generating the random numbers from the distribution. Algorithm for sample generation from the EILD is given by

\section{Algorithm 1:}

Step 1. Set $n, \alpha, \& \theta$.

Step 2. Generate an inverse gamma random variable, $V_{j} \sim I G(\alpha-1, \theta)$ for $j=1,2, \ldots, n$.

Step 3. Generate an inverse gamma random variable, $W_{j} \sim I G(\alpha, \theta)$ for $j=1,2, \ldots, n$.

Step 4. Generate a Uniform random variable, $U_{j} \sim U(0,1)$ for $j=1,2, \ldots, n$.

Step 5. If $U_{j} \leq(\theta /(\theta+1))$, then set $X_{i}=V_{i}$, otherwise, set $X_{i}=W_{i}$ for $j=1,2, \ldots, n$.

On the basis of simulated samples, we study the behaviour of biases, absolute biases and mean squared error (MSE) of MLEs of $\alpha$ and $\theta$ with varying sample size $n$. We also investigated the coverage probability for the asymptotic confidence intervals. Simulation algorithm consists the following steps:

\section{Algorithm 2:}

Step 1. Generate 10,000 samples of size $n$ from the $\operatorname{EILD}(2,2)$ by using the Algorithm 1.

Step 2. Compute the MLEs for the 10,000 samples,say $\hat{\alpha}_{i}, \hat{\theta}_{i}$ for $\mathrm{i}=1,2, \ldots, 10,000$.

Step 3. Compute the standard errors of the MLEs for the 10,000 sample say $s_{\hat{\alpha}_{i}}, s_{\hat{\theta}_{i}}$. The standard errors were computed by inverting the observed information matrices.

Step 4. Compute average estimate (AE) as

$$
A E(\hat{\Theta})=\frac{1}{n} \sum_{i=1}^{n} \hat{\Theta}_{i}
$$

Step 5. Compute the biases, absolute biases (AB) and mean squared error (MSE) using the following formulae,

$$
\begin{gathered}
B_{\Theta}(n)=\frac{1}{10,000} \sum_{i=1}^{10,000}\left(\hat{\Theta}_{i}-\Theta\right) \\
A B_{\Theta}(n)=\frac{1}{10,000} \sum_{i=1}^{10,000}\left|\left(\hat{\Theta}_{i}-\Theta\right)\right| \\
M S E_{\Theta}(n)=\frac{1}{10,000} \sum_{i=1}^{10,000}\left(\hat{\Theta}_{i}-\Theta\right)^{2}
\end{gathered}
$$

for $\Theta=\{\alpha, \theta\}$. 


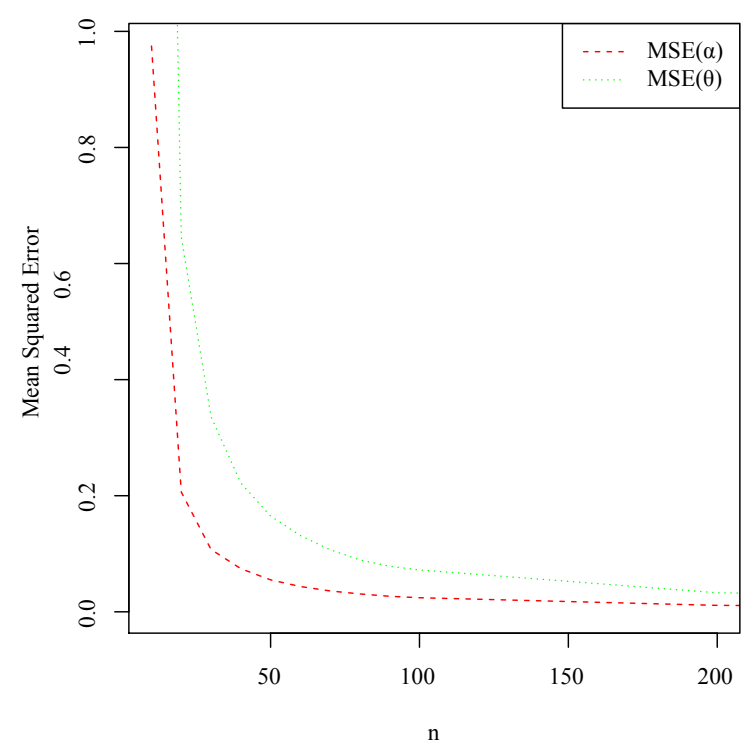

(a) MSE.

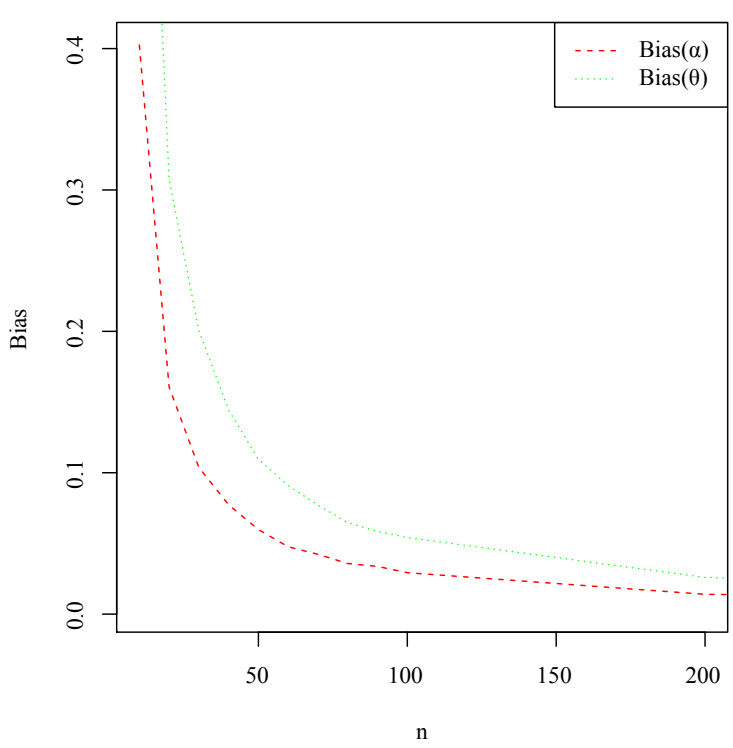

(b) Biasedness.

Figure 4: Mean squared error (MSE) and Biasedness of $\hat{\alpha}$ an $\hat{\theta}$ with varying $n$.

Step 6. Compute the coverage probabilities (CP) and coverage widths (CW) given by

$$
\begin{gathered}
\mathrm{CP}_{\Theta}(\mathrm{n})=\frac{1}{10,000} \sum_{1=1}^{10,000} \mathrm{I}\left(\hat{\Theta}_{1}-1.959964 \mathrm{~s} \hat{\Theta}_{1} \leq \Theta \leq \hat{\Theta}_{1}+1.959964 \mathrm{~s}_{\hat{\Theta}_{1}}\right) \\
\mathrm{CW}_{\Theta}(\mathrm{n})=\frac{3.919928}{10,000} \sum_{i=1}^{10,000} \mathrm{~s}_{\hat{\Theta}_{1}}
\end{gathered}
$$

for $\Theta=\{\alpha, \theta\}$, where I (.) denotes the indicator function.

We repeat these steps for $\mathrm{n}=10,20,30,40,50,60,70,80,90,100,200,500,1000$ with $\alpha=2$ and $\theta$ $=2$, and computed $\operatorname{Bias}_{\Theta}(\mathrm{n}), \mathrm{MSE}_{\Theta}(\mathrm{n}), \mathrm{CP}_{\Theta}(\mathrm{n})$ and $\mathrm{CW}_{\Theta}(\mathrm{n})$. Trends for the results have also been shown graphically in Figures 4, 5, 6. From the results, it can be observed that the biases and MSEs of the MLEs of $\alpha$ and $\theta$ decrease as sample size $n$ increases. That proves the consistency of the MLEs. In general, the CP for the CIs of $\alpha$ and $\theta$ decrease with increasing sample size. However, it stabilizes at approximately 0.95 for large sample size. The average width of CIs for $\alpha$ and $\theta$ decreases as sample size increase.

\section{Real data analysis}

In this section, we use a set of real data (given in table 1) of flood levels to demonstrate the applicability of the extended inverse Lindley distribution. The data were obtained in a civil engineering context and gives the maximum flood level (in millions of cubic feet per second) for the Susquehanna river at Harrisburg, Pennsylvania over 20 four-year periods from 1890 to 1969 . These data have been widely discussed by many authors and were initially reported by Dumonceaux and Antle (1973). First, we use the likelihood ratio (LR) test statistic to check 


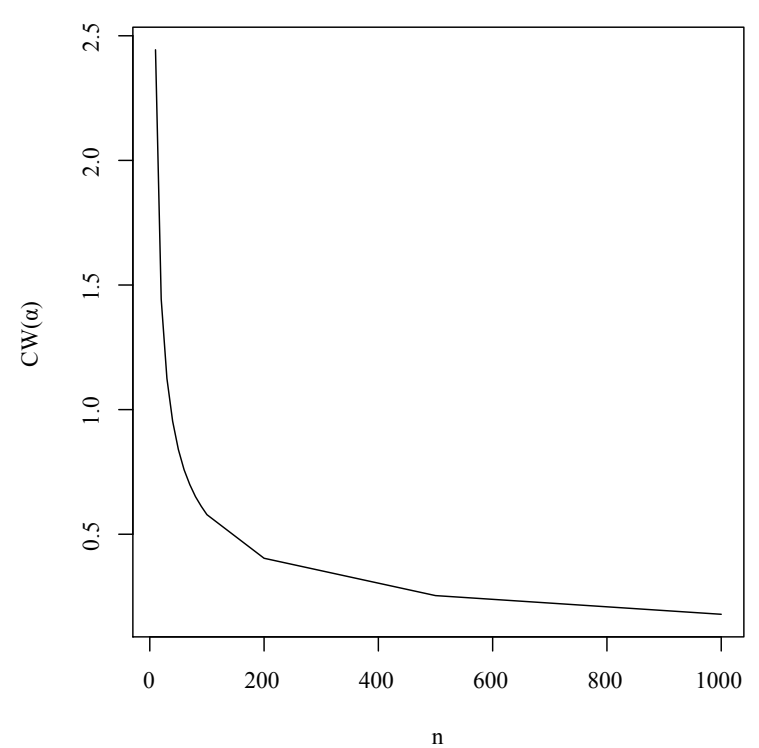

(a) For $\alpha$.

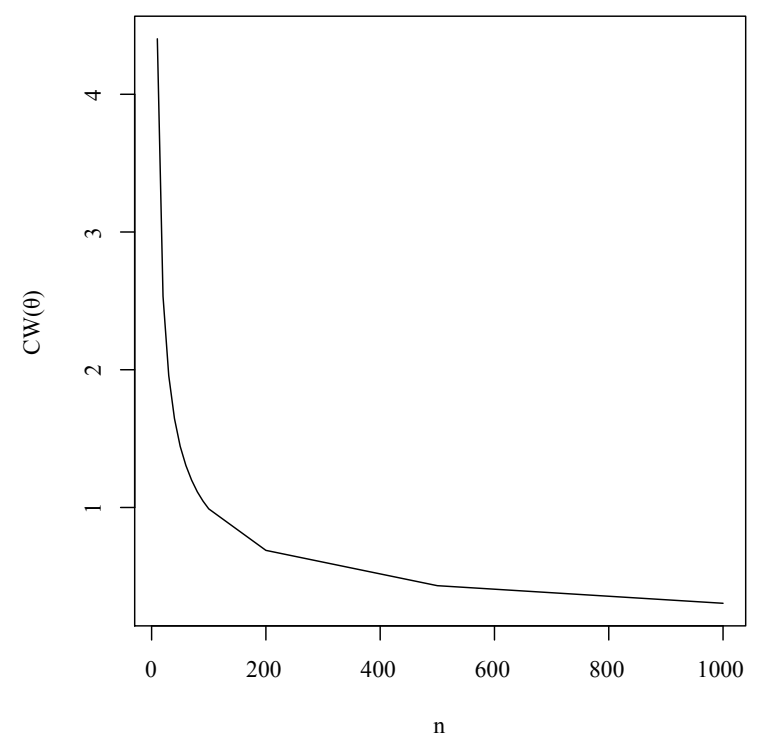

(b) For $\theta$.

Figure 5: Coverage width of asymptotic confidence intervals of $\hat{\alpha}$ an $\hat{\theta}$ with varying $n$.

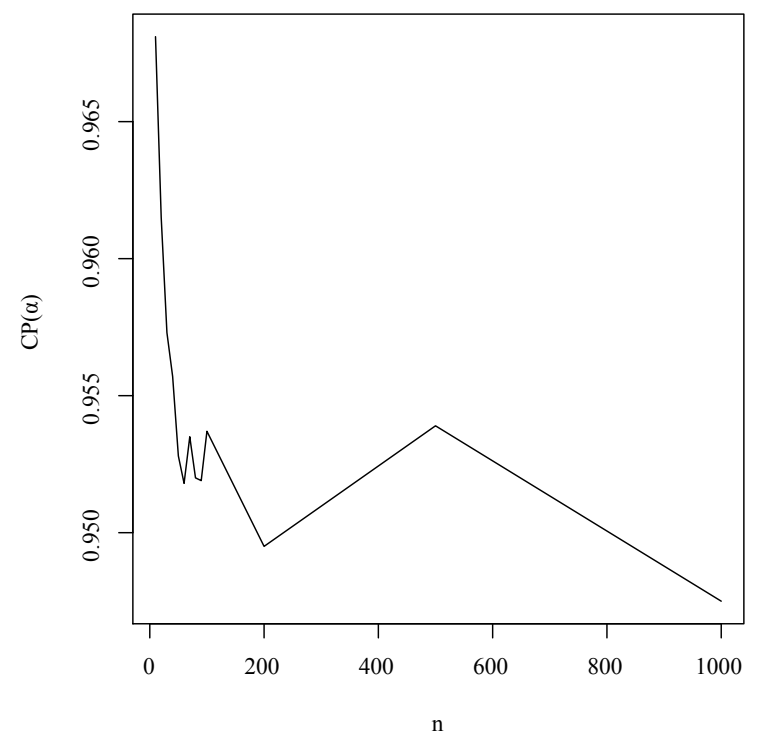

(a) For $\alpha$.

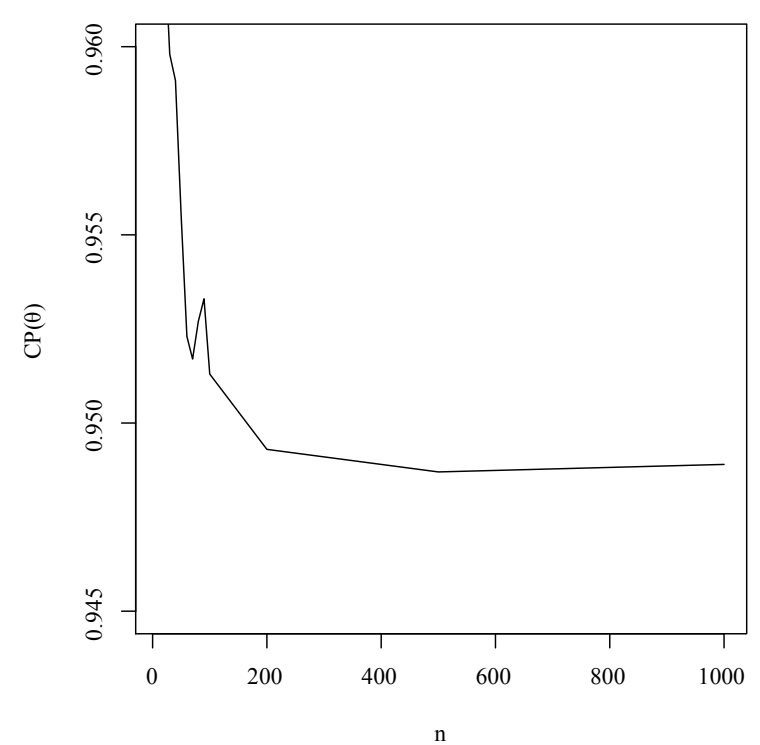

(b) For $\theta$.

Figure 6: Coverage probability of asymptotic confidence intervals of $\hat{\alpha}$ an $\hat{\theta}$ with varying $n$. Table 1 :

Flood level data

\begin{tabular}{rrrrr}
\hline 0.654 & 0.654 & 0.315 & 0.449 & 0.297 \\
0.324 & 0.269 & 0.74 & 0.418 & 0.412 \\
0.338 & 0.392 & 0.484 & 0.265 & 0.379 \\
0.423 & 0.379 & 0.402 & 0.494 & 0.416 \\
\hline
\end{tabular}


whether the shape parameter associated with extended inverse Lindley distribution improves its applicability. The hypothesis can be stated as

$$
\begin{gathered}
\text { null hypothesis; } H_{0}: \mathrm{X} \sim \operatorname{ILD}(\theta)(\text { i.e. } \alpha=1) \\
\text { versus } \\
\text { alternative hypothesis; } H_{1}: \mathrm{X} \sim \operatorname{EILD}(\alpha, \theta)(\text { i.e. } \alpha \neq 1) .
\end{gathered}
$$

In this case, the LR test statistic for testing $H_{0}$ versus $H_{1}$ is $\zeta\left(l_{0}-l_{1}\right)$, where $l_{1}$ and $l_{0}$ are the log-likelihood functions under $H_{1}$ and $H_{0}$, respectively. The statistic $\zeta$ is asymptotically $($ as $n \rightarrow \infty)$ distributed as $\chi_{k}^{2}$ with $\mathrm{k}$ degree of freedom, where $\mathrm{k}$ is the number of parameters. The LR test rejects $H_{0}$ if $\zeta \geq \chi_{k}^{2}(\gamma)$, where $\chi_{k}^{2}(\gamma)$ denotes the upper $100 \gamma \%$ quantile of the $\chi_{k}^{2}$ distribution. For given real data set, the log-likelihood under the ILD is $l_{0}=0.5854$ with $\hat{\theta}=0.6344$ and under $H_{1}, l_{1}$ is 16.1422 with $(\hat{\alpha}=15.556, \hat{\theta}=5.7875)$. Clearly, the shape parameter $\alpha$ can never be 1 for the data since $\hat{\alpha}=15.556$ which is very far than unity. However, the LR test statistic is $\zeta=31.11$ which is greater than $\chi_{1}^{2}(0.05)=3.84$. The results indicate that evidences do not support the null hypothesis. Therefore, the EILD is a better model than its special case, ILD.

Further, we also applied another statistical tools such as Kolmogorov-Smirnov (KS) statistic, Akaike information criterion (AIC) and Bayesian information criterion (BIC), to choose the best possible model for the data set among all competitive models. For this purpose, we consider seven alternative distribution such as inverse Weibull $(\operatorname{IW}(\alpha, \theta))$, inverse gamma $(\operatorname{IGM}(\alpha, \theta))$, generalised inverse exponential $(\operatorname{GIE}(\alpha, \theta))$, inverse Gaussian $(\operatorname{IG}(\alpha, \theta))$, lognormal $\left(\operatorname{LN}\left(\mu, \sigma^{2}\right)\right), \log$-Logistic $(\operatorname{LLog}(\alpha, \beta))$ and flexible Weibull $(\mathrm{FW}(\alpha, \theta))$. The statistical tools used are described as follows: KS test statistic, $D_{n}=\sup _{x}\left|F(x)-F_{n}(x)\right|, \mathrm{AIC}=-2 \log L+2 p$, and $\mathrm{BIC}=-2 \log L+p \log n$, where, $p$ is the number of parameters are to be estimated from the data.

The selection criterion is that the lowest AIC and BIC correspond to the best model fitted. The MLEs, AIC and BIC are shown in Table 2. From the Table, we can observed that the extended inverse Lindley distribution shows the smaller AIC and BIC than other competing distributions. Thus, the EILD fits well the data set. The plots of probability-probability and the fitted cumulative distribution of the EILD are shown in Figure 7 for maximum flood level data. Figures also indicate that the EILD is a good fitted model for the data. The fitted density of EILD is shown in Figure 8 .

Table 2: MLEs, AIC, BIC and KS for the fitted models based on real data.

\begin{tabular}{cccccc}
\hline Model & MLE & LogL & AIC & BIC & KS \\
\hline $\operatorname{EILD}(\hat{\alpha}, \hat{\theta})$ & $\hat{\alpha}=15.556, \hat{\theta}=5.7875$ & 16.1422 & -32.2845 & -26.2930 & 0.1271 \\
$\operatorname{IW}(\hat{\alpha}, \hat{\theta})$ & $\hat{\alpha}=4.3140, \hat{\theta}=0.0119$ & 16.0974 & -32.1947 & -26.2033 & 0.1060 \\
$\operatorname{IGM}(\hat{\alpha}, \hat{\theta})$ & $\hat{\alpha}=14.578, \hat{\theta}=5.7383$ & 16.1416 & -32.2833 & -26.2918 & 0.1272 \\
$\operatorname{GIE}(\hat{\alpha}, \hat{\theta})$ & $\hat{\alpha}=37.7001, \hat{\theta}=1.645$ & 15.4112 & -30.8224 & -24.8309 & 0.1584 \\
$\operatorname{IG}(\hat{\alpha}, \hat{\theta})$ & $\hat{\alpha}=0.4231, \hat{\theta}=5.6410$ & 15.8520 & -31.7040 & -25.7126 & 0.1469 \\
$\operatorname{LN}\left(\hat{\mu}, \hat{\sigma}^{2}\right)$ & $\hat{\mu}=-0.897, \hat{\sigma}^{2}=0.269$ & 15.8242 & -31.6484 & -25.6570 & 0.2843 \\
$\operatorname{LLog}(\hat{\alpha}, \hat{\beta})$ & $\hat{\alpha}=0.4018, \hat{\beta}=6.5752$ & 15.6859 & -31.3717 & -25.3803 & 0.9999 \\
$\operatorname{FW}(\hat{\alpha}, \hat{\theta})$ & $\hat{\alpha}=3.6456, \hat{\theta}=0.8217$ & 13.5886 & -27.1773 & -21.1858 & 0.3149 \\
\hline
\end{tabular}




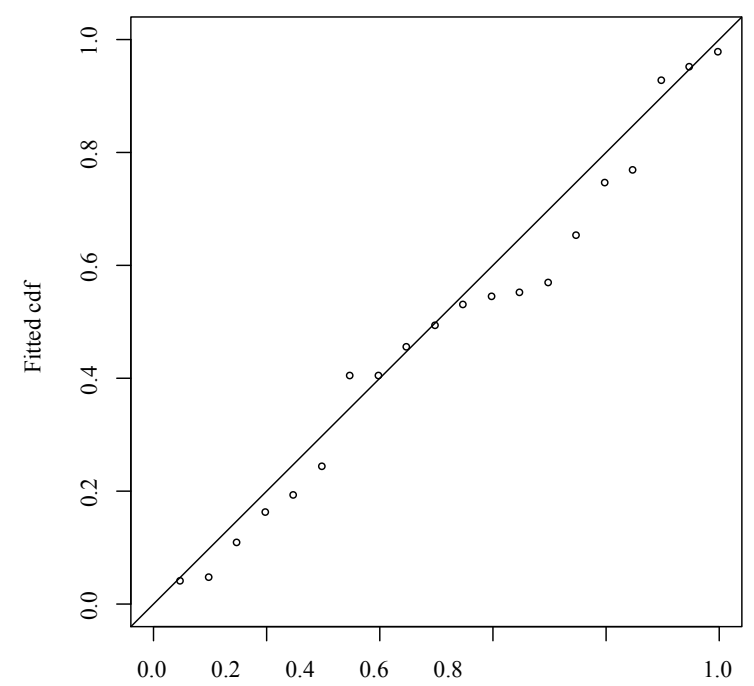

Probabilit y-probability (PP) plot.

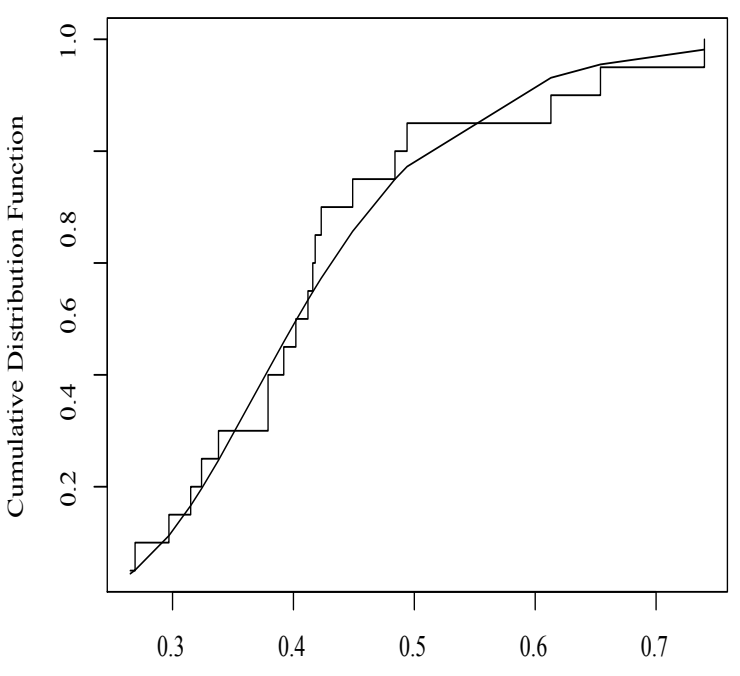

Cumulativ e distribution function.

Figure 7: Probability-probability (PP) and fitted cdf of EILD based on real data.

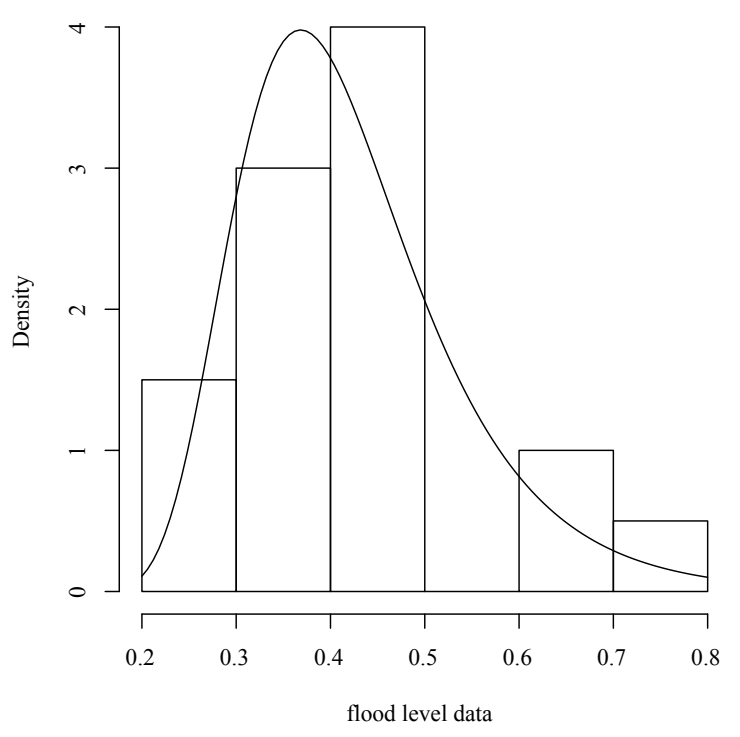

Figure 8: Plot of the fitted density of the EILD for real data. 


\section{Summary and conclusion}

In this article, we introduced two parameter extension of inverse Lindley distribution, called extended inverse Lindley distribution (EILD). The distribution shows the upside-down bathtub shape for its hazard rate. The current extension provides a rather general and flexible framework for statistical analysis of positive data. The EILD can be expressed as a two component mixture of the inverse gamma distributions. That provides some expansions for the ordinary moments, mean deviations, stochastic ordering, inverse moments and Renyi entropy. The estimation of parameters is approached by the method of maximum likelihood. The confidence intervals for the parameters of EILD are also obtained by using asymptotic distribution of MLEs. Simulation study has been carried out to study the behaviours of MLEs of the parameters with respect to sample size. We considered the likelihood ratio test statistic to compare the model with its baseline model. An application of the EILD shows that it could provide a better fit than other alternative inverse distributions. Finally, we hope that our proposed model will attain great applicability in the real problems encountered in various disciplines such as medical, engineering and social sciences etc.

\section{Acknowledgements}

The authors would like to thank the reviewers and the editors for their comments for the improvement of the paper. Miss Pragya Khandelwal thanks to the Department of Statistics, CURAJ, for proving all necessary laboratory facilities for this project.

\section{References}

[1] Abouammoha, A.M., Alshangitia, A.M., Ragabb, I.E., 2015. A new generalized Lindley distribution. Statistical Computation and Simulation DOI:10.1080/00949655.2014.995101.

[2] Akmakyapan, S., Kadlar, G.Z., 2014. A new customer lifetime duration distribution: The Kumaraswamy Lindley distribution. International Journal of Trade, Economics and Finance 5, 441-444.

[3] Al-Mutairi, D.K., Ghitany, M.E., Kundu, D., 2013. Inferences on stress-strength reliability from Lindley distributions. Communications in Statistics - Theory and Methods 42, 1443-1463.

[4] Alkarni, S.H., 2015. Extended inverse lindley distribution: properties and application. Springer- Plus $4,1-13$.

[5] Ashour, S.K., Eltehiwy, M.A., 2015. Exponentiated power Lindley distribution. Journal of Advanced Research 6, 895-905.

[6] Dumonceaux, R., Antle, C., 1973. Discrimination between the lognormal and weibull distribution. Technometrics 15, 923-926.

[7] Elbatal, I., Elgarhy, M., 2013. Statistical properties of Kumaraswamy quasi Lindley distribution. International Journal of Mathematics Trends and Technology 4, 237-246.

[8] Ghitany, M., Al-Mutairi, D., Balakrishnan, N., Al-Enezi, L., 2013. Power Lindley distribution and associated inference. Computational Statistics and Data Analysis 64, 20-33.

[9] Ghitany, M., Atieh, B., Nadarajah, S., 2008. Lindley distribution and its application. Math. Comput. Simul. 78, 493-506.

[10] Krishna, H., Kumar, K., 2011. Reliability estimation in Lindley distribution with progressively type-ii right censored sample. Math. Comput. Simul. 82, 281-294.

[11] Lindley, D., 1958. Fiducial distributions and bayes theorem. J. R. Statist. Soc. Ser. B 20, 102-107.

[12] Mahmoudi, E., Zakerzadeh, H., 2010. Generalized poisson-Lindley distribution. Communications in Statistics - Theory and Methods 39, 1785-1798. 
[13] Mazucheli, J., Achcar, J.A., 2011. The Lindley distribution applied to competing risks lifetime data. Computer Methods and Programs in Biomedicine 104, 188-192.

[14] Merovci, F., 2013. Transmuted Lindley distribution. Int. J. Open Problems Compt. Math. 6, 63-72.

[15] Merovci, F., Elbatal, I., 2014. Transmuted Lindley-geometric distribution and its applications.J. Stat. Appl. Pro. 3, 77-91.

[16] Merovci, F., Sharma, V.K., 2014. The beta Lindley distribution: Properties and applications.Journal of applied mathematics 2014, 1-10.

[17] Nadarajah, S., Bakouch, H., Tahmasbi, R., 2011. A generalized Lindley distribution. Sankhya B - Applied and Interdisciplinary Statistics 73, 331-359.

[18] Oluyede, B.O., Yang, T., 2015. A new class of generalized Lindley distributions with applications. Journal of Statistical Computation and Simulation 85, 2072-2100.

[19] Renyi, A., 1961. On measures of entropy and information, in: Proceedings of the 4th Berkeley Symposium on Mathematical Statistics and Probability, University of California Press, Berkeley. pp. 547-561.

[20] Shaked, M., Shanthikumar, J., 1994. Stochastic orders and their applications. Boston: Academic Press.

[21] Sharma, V.K., Singh, S.K., Singh, U., 2014a. A new upside-down bathtub shaped hazard rate model for survival data analysis. Applied Mathematics and Computation 239, 242-253.

[22] Sharma, V.K., Singh, S.K., Singh, U., Agiwal, V., 2015. The inverse Lindley distribution: A stress reliability model with application to head and neck cancer data. Journal of Industrial and Production Engineering 32, 162-173.

[23] Sharma, V.K., Singh, S.K., Singh, U., Merovci, F., 2014b. The generalized inverse Lindley distribution: A new inverse statistical model for the study of upside-down bathtub data. Com- munications in Statistics-Theory and Methods In press, DOI:10.1080/03610926.2014.948206.

[24] Singh, B., Gupta, P.K., 2012. Load-sharing system model and its application to the real data set. Mathematics and Computers in Simulation 82, 1615-1629.

[25] Zakerzadeh, H., Dolati, A., 2009. A generalised Lindley distribution. Journal of math extension 3, 13-25. 